\title{
HOMOLOGY OF COMPLEX PROJECTIVE HYPERSURFACES WITH ISOLATED SINGULARITIES
}

\author{
JOHN L. MILLER ${ }^{1}$
}

\begin{abstract}
We use information concerning the homology of links and Milnor fibers at each singularity of a hypersurface $\hat{\nabla}$, of degree $d$ in $C P^{n+1}$ with only isolated singularities, to determine $\bar{V}$ s .homology except for the question of torsion in $H_{n}(\tilde{V} ; Z)$.
\end{abstract}

Consider a projective hypersurface $\tilde{V}$ of degree $d$ in $C P^{n+1}$ having only isolated singularities $P=\left\{p_{1}, \ldots, p_{m}\right\}$. Results of Howard [3] and Andreotti and Frankel [1] imply that we may regard $\tilde{V}$ as the closure of an affine hypersurface $V=f^{-1}(0) \subset C^{n+1}$ where $f: C^{n+1} \rightarrow C$ is a polynomial of degree $d$ such that

(1) $P \subset V$,

(2) $\tilde{V}$ is nowhere tangent to the hyperplane at infinity:

$$
H_{0}=\left\{\left[z_{0}: z_{1}: \cdots: z_{n+1}\right] \in C P^{n+1} \mid z_{0}=0\right\} .
$$

Given this situation we note that for sufficiently small $\varepsilon>0$ the projective completion $\tilde{V}_{\varepsilon}$ of the affine hypersurface $V_{\varepsilon}=f^{-1}(\varepsilon)$ must be nonsingular. Furthermore $\tilde{V}_{\varepsilon} \cap H_{0}=\tilde{V} \cap H_{0}$. By pushing along the vector field obtained by integrating $-\operatorname{grad}_{C} f=\left\langle-\overline{\partial f} / \partial Z_{1}, \ldots,-\overline{\partial f} / \partial Z_{n+1}\right\rangle$ in $C^{n+1}$ we obtain a map $\Phi: V_{\varepsilon} \rightarrow V$ and since $\tilde{V}$ is transverse to $H_{0}$ this extends to $\tilde{\phi}: \tilde{V}_{\varepsilon} \rightarrow \tilde{V}$ by defining $\tilde{\phi} \mid \tilde{V}_{\varepsilon} \cap H_{0}$ to be the identity. For $i=1, \ldots, m$ and $\delta>0$, let $S_{\delta, i}$ be a $(2 n+1)$-sphere of radius $\delta$ centered at $p_{i} \in C^{n+1}$, also $K_{\delta, i}=S_{\delta, i} \cap V$. Choose $\delta_{1}>0$ small enough so that for $\delta \leqslant \delta_{1}$, we get a Milnor fibration $F_{i} \rightarrow S_{\delta, i} i K_{\delta, i} \stackrel{\theta_{i}}{\rightarrow} S^{1}$. Here $\theta_{i}(Z)=f(z) /|f(z)|$. Let $\delta_{0}<\delta_{1}$ and $D_{i, 0}\left(D_{i, 1}\right)$ be the open $2 n$-disk with radius $\delta_{0}\left(\delta_{1}\right)$, respectively, around $p_{i}$.

THEOREM 1. Letting $D_{1}=\cup_{i=1}^{m} D_{i, 1}$ and $D_{0}=\cup_{i=1}^{m} D_{i, 0}$, $\tilde{\phi} \mid\left(\tilde{\tilde{V}_{\varepsilon}} \mid D_{0}\right)$ is isotopic to a diffeomorphism $\left(\tilde{V_{\varepsilon}} D_{0}\right) \approx\left(\tilde{V} \backslash D_{0}\right)$ for sufficiently small $\varepsilon>0$.

Proof. Choose $\varepsilon$ small enough so that

(1) $V_{\varepsilon} \cap D_{i, 0} \approx F_{i}$ the Milnor fiber at $p_{i}$; see [6, Lemma 5.11].

(2) $f^{-1}(\gamma)$ is nonsingular for $0<|\gamma|<\varepsilon$.

(3) $\phi^{-1}\left[p_{i}\right] \subset V_{\varepsilon} \cap D_{i, 0}$ thus insuring that $\phi$ is $1-1$ on $V_{\varepsilon} \backslash D_{0}$.

The fact that $f^{-1}(\gamma)$ is transverse to $S_{\delta, i}$ for $0<|\gamma| \leqslant \varepsilon$ and $\delta_{0} \leqslant \delta \leqslant \delta_{1}$

Received by the editors June 17, 1975 and, in revised form, November 18, 1975.

AMS (MOS) subject classifications (1970). Primary 57A65, 14M99; Secondary 32C40.

Key words and phrases. Hypersurface, link, Milnor fiber.

1 The results of this paper are contained in the author's doctoral dissertation prepared at the University of California (Berkeley) under the direction of Professors P. Emery Thomas and Alan H. Durfee. 
means that the component of $-\operatorname{grad}_{C} f$ which is tangent to $S_{\delta}$ is nonvanishing. Hence we may obtain a new vector field by using a partition of unity in the collar $D_{1} \backslash D_{0}$ to smoothly patch $-\operatorname{grad}_{C} f$ in $f^{-1}[0, \varepsilon] \backslash D_{1}$ with the tangential component of $-\operatorname{grad}_{C} f$ on $X_{0}$. Pushing along this vector field yields the diffeomorphism $V_{\varepsilon} \backslash D_{0} \approx V \backslash D_{0}$. By smoothly altering the partition of unity, one shows this diffeomorphism to be isotopic to $\phi: V_{\varepsilon} \backslash D_{0} \rightarrow V \backslash P$. Taking projective completions and defining the levels of the isotopy to be the identity on $\tilde{V}_{\varepsilon} \cap H_{0}$ completes the theorem.

COROLlaRY 2. Letting $W$ denote a nonsingular hypersurface of degree $d$ in $C P^{n+1}$ we have the following cohomology ladder (Z-coefficients).

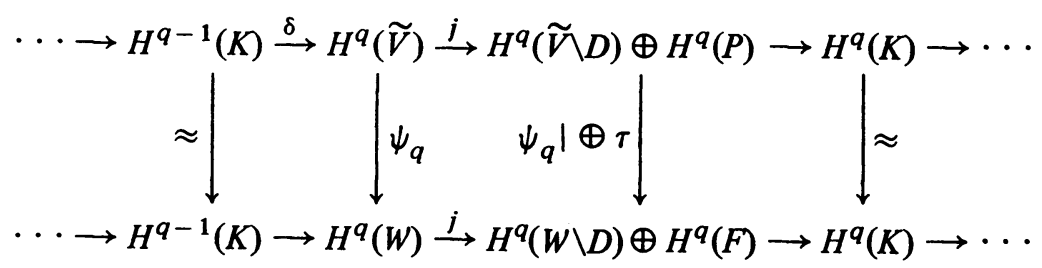

Here $F$ is the disjoint union of the Milnor fibers at each point of the singular locus $P=\left\{p_{1}, \ldots, p_{m}\right\}, K=\delta F$, and $\psi_{q} \mid: H^{q}(\tilde{V} \backslash D) \rightarrow H^{q}(W \backslash D)$ is an isomorphism for all $q$.

Proof. Consider the Mayer-Vietoris sequence resulting from the following adjunctions:

(i) $\tilde{V}=\left(\tilde{V} \backslash D_{0}\right) \cup K_{K_{0}}$ Cones on $K_{i, 0}$;

(ii) $W=\tilde{V}_{\varepsilon} \approx\left(\tilde{V}_{\varepsilon} \mid D_{0}\right) \cup \cup_{0} \bar{F}$, where $\bar{F}=\cup \bar{F}_{i}$ and $\bar{F}_{i}=D_{0} \cap \tilde{V}_{\varepsilon}$ and is diffeomorphic to the closed Milnor fiber at $p_{i}$.

The vertical maps are induced by $\tilde{\phi}$ and since $H^{q}(P)=0$ for $q \geqslant 1, \tau$ is the zero map; for $q=0, \tau$ is an isomorphism. $\tilde{\psi}_{q} \mid$ is an isomorphism by Theorem 1.

THEOREM 3. If $\tilde{V}$ is a projective hypersurface $\subset C P^{n+1}$ of degree $d$ with singular locus $P=\left\{p_{1}, \ldots, p_{m}\right\}$, then

(i)

$$
H^{q}(\tilde{V} ; Z)= \begin{cases}Z, & \text { q even } \neq n, n+1, \\ 0, & q \text { odd } \neq n, n+1,\end{cases}
$$

(ii) Free $H^{n+1}(\tilde{V} ; Z)$ has rank

$$
\left\{\begin{array}{l}
1+\sum_{1}^{m} \tilde{\beta}_{n-1}\left(K_{i}\right), \quad n \text { odd }, \\
\sum_{1}^{m} \tilde{\beta}_{n-1}\left(K_{i}\right), \quad n \text { even } ;
\end{array}\right.
$$

(iii) $H^{n}(\tilde{V} ; Z)$ is free with rank $\beta_{n}(W)-\sum_{1}^{m} \mu_{i}+\sum_{1}^{m} \tilde{\beta}_{n-1}\left(K_{i}\right)$ where $\beta_{n}(W)=\operatorname{rank} H^{n}(W)$, and is well known to equal

$$
\left\{\begin{array}{l}
d^{-1}(d-1)\left[(d-1)^{n+1}-1\right], \quad n \text { odd }, \\
d^{-1}\left[(d-1)^{n+2}-1\right]+2, \quad n \text { even, }
\end{array}\right.
$$


and $\mu_{i}=H^{n}\left(F_{i}\right) . \tilde{\beta}_{n-1}\left(K_{i}\right)$ denotes the reduced rank of $H^{n-1}\left(K_{i}\right)$.

(iv) $\delta \mid:$ Tor $H^{n}(K ; Z) \rightarrow$ Tor $H^{n+1}(\tilde{V} ; Z)$ is epic.

Proof. Statements (i), (ii), and (iii) follow from the facts that $K$ is an $(n-1)$-connected $(2 n-1)$-manifold, $F$ is a $2 n$-manifold with $\partial F=K$ and is of the same homotopy type as a wedge of $S^{n}$ 's, and the cohomology ladder of Corollary 2. Suppose $x \in H^{n}(\tilde{V} ; Z)$ and $m x=0$. Hence $m \cdot \psi_{n}(x)=0$ and, since $H^{n}(W ; Z)$ is free, $\psi_{n}(x)=0$. Thus $j \cdot \psi_{n}(x)=\left(\psi_{n} \mid \oplus \tau\right) \cdot j(x)=0$. But $H^{n}(P ; Z)=0$ and $\psi_{n} \mid$ is an isomorphism. Hence $j(x)=0$ and $\exists y$ $\in H^{q-1}(K)$ such that $\delta(y)=x$ by exactness.

\section{BIBLIOGRAPHY}

1. A. Andreotti and T. Frankel, The second Lefschetz theorem on hyperplane sections, Global Analysis (Papers in Honor of K. Kodaira), Univ. of Tokyo Press, Tokyo, 1969, pp. 1-20. MR 42 \#5989.

2. F. Hirzebruch, W. Neumann and S. Koh, Differentiable manifolds and quadratic forms, Dekker, New York, 1971. MR 49 \#6250.

3. A. Howard, On the homotopy groups of an affine algebraic hypersurface, Ann. of Math. (2) 84 (1966), 197-216. MR 35 \#4946.

4. M. Kato and Y. Matsumoto, Simply connected surgery of submanifolds in codimension two. I (preprint).

5. J. Milnor, Morse theory, Ann. of Math. Studies, no. 51, Princeton Univ. Press, Princeton, N. J., 1963. MR 29 \#634.

6. Singular points of complex hypersurfaces, Ann. of Math. Studies, no. 61, Princeton Univ. Press, Princeton, N. J.; Univ. of Tokyo Press, Tokyo, 1968. MR 39 \#969.

7. J. Milnor and P. Orlik, Isolated singularities defined by weighted homogeneous polynomials, Topology 9 (1970), 385-393. MR 45 \#2757.

8. E. Thomas and J. Wood, On manifolds of representing homology classes in codimension 2, Invent. Math. 25 (1974), 63-89.

Department of Mathematics, University of Hawail, Honolulu, Hawail 96822 